\title{
Optimizing Reverse Osmosis Membrane Parameters through the Use of the Solution-Diffusion Model: A Review
}

\author{
Farah Z. Najdawi, Kaleb T. Neptune \\ Department of Material Science Engineering, and Commercialization, Texas State University, San Marcos, TX, USA \\ Email: fzn1@txstate.edu
}

How to cite this paper: Najdawi, F.Z. and Neptune, K.T. (2022) Optimizing Reverse Osmosis Membrane Parameters through the Use of the Solution-Diffusion Model: A Review. Engineering, 14, 9-32.

https://doi.org/10.4236/eng.2022.141002

Received: November 8, 2021

Accepted: January 4, 2022

Published: January 7, 2022

Copyright $\odot 2022$ by author(s) and Scientific Research Publishing Inc. This work is licensed under the Creative Commons Attribution International License (CC BY 4.0).

http://creativecommons.org/licenses/by/4.0/

\begin{abstract}
When designing and building an optimal reverse osmosis (RO) desalination plant, it is important that engineers select effective membrane parameters for optimal application performance. The membrane selection can determine the success or failure of the entire desalination operation. The objective of this work is to review available membrane types and design parameters that can be selected for optimal application to yield the highest potential for plant operations. Factors such as osmotic pressure, water flux values, and membrane resistance will all be evaluated as functions of membrane parameters. The optimization of these parameters will be determined through the deployment of the solution-diffusion model devolved from the Maxwell Stephan Equation. When applying the solution-diffusion model to evaluate RO membranes, the Maxwell Stephan Equation provides mathematical analysis through which the steps for mass transfer through a RO membrane may be observed and calculated. A practical study of the use of the solution-diffusion model will be discussed. This study uses the diffusion-solution model to evaluate the effectiveness of a variety of Toray RO membranes. This practical application confirms two principal hypotheses when using the diffusion-solution model for membrane evaluation. First, there is an inverse relationship between membrane and water flux rate. Second, there is a proportional linear relationship between overall water flux rate and the applied pressure across a membrane.
\end{abstract}

\section{Keywords}

Reverse Osmosis Membrane, Solution-Diffusion Model, Maxwell Stephan Equation, Desalination Plants, Membrane Optimization

\section{Introduction}

Understanding the physical parameters of a reverse osmosis membrane can help 
engineers optimize desalination plant production. The optimization of plant production can help meet the global demand for clean water. Mathematical models can help resolve the optimization parameters of these systems which will allow for effective improvements. Any effort that improves clean water production inevitably contributes to the global demand for clean water which indirectly betters the quality of life for many. This review analyzes developments in membrane design and membrane parameters in order to illuminate the optimal parameters with the solution-diffusion model. First, an overview of reverse osmosis systems will be provided, followed by an overview of the membrane structures used in reverse osmosis. Next, the motivation for employing reverse osmosis systems is outlined, followed by models for exploring mass transfer through membranes. Then, the solution-diffusion model is examined in relation to how it can help us optimize RO membrane parameters. Developments in thin, highly permeable membranes are analyzed next, and then, finally, this review explores practical applications of the solution-diffusion model relative to $\mathrm{RO}$ membrane construction.

\section{Reverse Osmosis Systems Overview}

Reverse Osmosis (RO) is a method of purifying water using a semi-permeable membrane which collects and removes ions, molecules, and some larger particles to produce clean drinking water [1]. In the process of reverse osmosis, osmotic pressure is overcome by an applied pressure, where osmotic pressure is a colligative property that is propelled by chemical potential difference between a solute and solvent. Reverse osmosis used for water treatment removes several types of dissolved and suspended species including bacteria from the water. Reverse osmosis is used in both industrial processes and clean potable water production. When reverse osmosis occurs, the solute is held on the side of the membrane that is pressurized, allowing the pure solvent to move through the membrane to the other side. This membrane is selective enough so as not to allow larger molecules or ions to pass through the pores. However, these pores should allow for smaller components like solvent molecules to move freely. In a typical osmotic process, the solvent will pass from the region of lower solute concentration (or higher water potential) through the membrane and to a region of lower solute concentration (or lower water potential). The drop in the free energy of the system is the force which propels the movement of the solvent when the difference in solvent concentration on either side of the membrane is reduced. This creates osmotic pressure from the solvent moving into the area of more concentrated solution. Reverse osmosis is the application of an external pressure which reverses the natural flow of a pure solvent. Because reverse osmosis utilizes diffusion, it can be reliant on pressure, flow rate, and other conditions that can be evaluated and optimized [2].

\section{A. Stages of RO treatment}

A desalination plant is composed of four primary systems as shown in Figure 1. These are the following: 1) pretreatment system; 2) high pressure; 3) pumps 


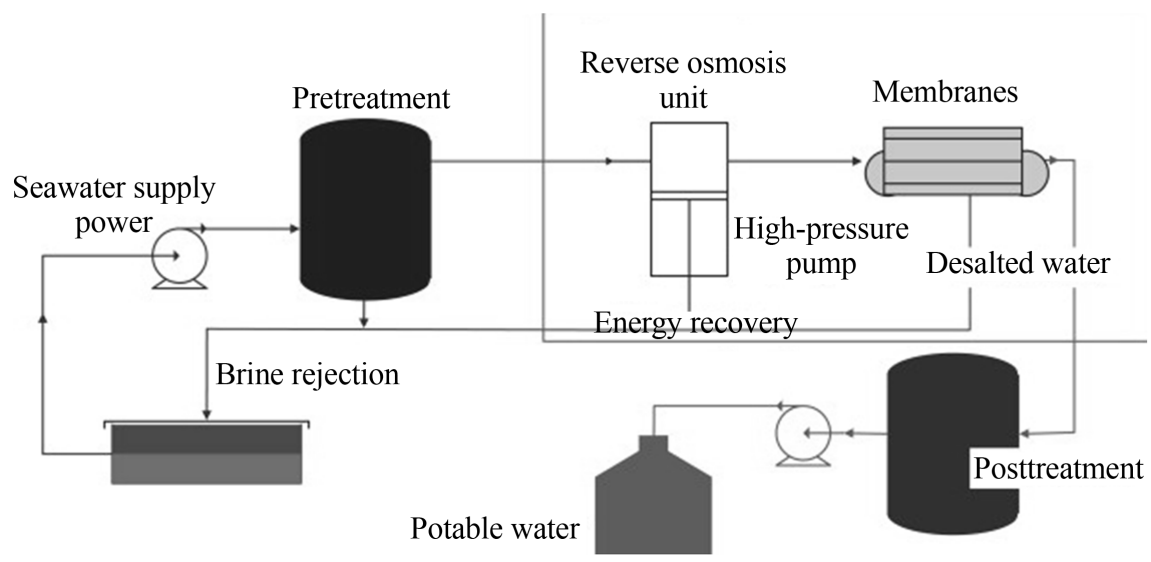

Figure 1. This diagram depicts the basic reserve osmosis system and subsystems [4].

and membrane systems; and 4) post-treatment. In the pretreatment system, all the suspended solids are removed so that salt precipitation or microbial growth can be prevented along the membranes. This process can involve conventional methods such as a chemical feed, often proceeded by coagulation/flocculation/sedimentation and sand filtration, or membrane feed processes like microfiltration and ultrafiltration. To achieve the pressure needed for the water to pass through the membrane and reject the salt, high-pressure pumps are used. After pretreatment, the water will travel to the pumped system where it will be pressurized to 17 to 27 bars for brackish water, and 52 to 69 bars for seawater [2]. Then the water will be forces through the RO membrane system. The membrane systems contain a pressure vessel and the semi-permeable membrane within. The water then will go through the post treatment process. Post-treatment can sometimes require adjusting the $\mathrm{pH}$ and disinfection depending on the quality of the water in the permeate and the use of the permeate [2].

In addition to the membranes, reverse osmosis systems also contain other methods of filtration, usually consisting of three, four, or five stages of filtration depending on the individual system. These filters are referred to as prefilters or postfilters based on if the water moves through them before or after it moves through the primary membrane. The sediment filter reduces particles development in the water, such as dust, dirt, and rust. The carbon filter reduces the presence of volatile organic compounds, chlorine, and other pollutants which would affect the water's taste or color. Finally, the semi-permeable membrane catches up to $98 \%$ of the total dissolved solids in the water [3].

\section{B. Advantages of RO systems}

There are eight principal advantages to employing reverse osmosis in processes such as dilute aqueous wastewater treatment. These are: 1) Reverse osmosis systems are relatively easy to design and use, including lower maintenance needs and modularity. RO systems can be expanded fairly easily; 2) The membrane filtration processes at work in reverse osmosis allow for the removal of organic and inorganic contaminants from the water; 3 ) The energy needs of reverse osmotic systems are lower than other water filtration methods; 4) Reverse osmosis sys- 
tems provide for the reclamation and recycling of waste process streams, which subsequently does not affect the quality of the material being recovered; 5) Reverse osmosis plants can typically be operated at ambient temperatures that reduce scale formation and corrosion problems, thus saving money in terms of maintenance and repair costs; 6) Since reverse osmosis systems possess a compartmental, modular structure, there is an increased flexibility with building desalination plants in a wide range of capacities; 7) Reverse osmotic systems enjoy a lower specific energy requirement; and 8) By using reverse osmosis processes, water treatment plants can significantly lower the volume of waste streams, allowing them to be treated in a more efficient and cost effective manner [2].

\section{Disadvantages of RO systems}

Household reverse osmosis units yield large amounts of water waste due to low back pressure and they only treat $5 \%$ to $15 \%$ of the water that enters the home. The rest will be purged as wastewater, and because this wastewater carries the unwanted pollutants, it is not common practice to recover this water with household systems. When wastewater is connected to the house drains it also adds to the load on the house's septic system. As an example, if a reverse osmosis system is producing five gallows of clean water a day, it can discharge anywhere between 20 and 90 gallons of wastewater per day. Another disadvantage of reverse osmosis system is that with their fine membrane construction, these systems can end up removing so many contaminants and foreign components from the water supply that desirable minerals that may be naturally occurring in the water are removed too. Some researchers have explored the ways in which our drinking of demineralized water might have long-term health effects [2].

\section{Applications of RO systems}

The process of reverse osmosis is inherently advantageous because of its membrane-based mechanism where concentration and separation can take place without a change of state and without the use of chemicals or thermal energy. This energy efficient characteristic makes reverse osmosis an ideal candidate for recovery applications. Reverse osmosis has been used for a wide array of applications and in an equally wide range of industries, including the beverage industry, spent wash from distilleries, groundwater treatment, recovery of phenol compounds, drinking water purification, hydrogen production, window cleaning, and even the reclamation of wastewater and seawater [5].

\section{R0 Membrane Structure Overview}

Reverse osmosis membranes are often characterized by their structure, material, geometric configuration, and the permeability. A variety of polymer materials are used but these membranes can be divided into two main groups based off their structures: conventional thin-film composites and thin-film nanocomposites. Conventional thin-film composites can be further classified into two main groups based off the materials used to make the membrane: Polyamide and cellulose acetate [6]. These membranes are also divided into three main groups 
based on the geometrical configuration of the membrane structure. These geomatical membrane configurations are hollow-fiber, spiral-wound, and flat-sheet. The permeability of the membrane is divided into three types: porous, non-porous, and dense.

\section{A. Membrane structures}

1) Conventional Thin-Film Composite Membrane Structure

The most widely used RO membranes are constructed of conventional thin-film composite membrane. These membranes are either made of aromatic polyamide (PA) or cellulose acetate (CA) thin films. The basic architecture of these membranes consists three layers. The first is the semipermeable PA or CA thin-film, typically only 0.2 micrometers thick, supported by a 0.025 to $0.05-\mathrm{mm}$ microporous layer. This microporous layer is cast on a third and final layer of reinforced fabric. The ultrathin polymeric film layer is the feature of this structure that gives the RO membrane the salt rejection capabilities [6]. The other two layers provide membrane reinforcement and help maintain integrity and durability of the structure. The semipermeable polymer film is constructed of a randomly oriented molecular structure with a high density and no porosity. Since this polymer has no porosity, the water molecules must transport though the membrane film by diffusion. Due to the random orientation of the molecular structure the path of the water molecules become multidimensional and curvilinear [6]. This is the fundamental reason that solution-diffusion is applied for analyzing the design of membrane structures.

2) Nanocomposite Membrane Structure

A novel membrane structure has been recently developed and provides evidence of higher specific permeability than conventional RO membranes at comparable salt rejection rates. These are thin-film nanocomposite membrane structures. Nanocomposite membranes are made of either a porous film consisting of an array of highly structured nanotubes densely and orderly packed or inorganic nanoparticles dispersed into the traditional membrane polymeric thin-film, PA or CA, membrane structures [7]. Nanocomposite membranes also have comparable or lower fouling rates than conventional thin-film composite RO membranes and can potentially be designed to remove specific ions. If these membranes evolve to a point where the entire membrane structure is made of tubes of uniform size, then these membranes could increase the water production capabilities by nearly $20 \%$ over conventional membrane structures [6].

\section{B. Membrane materials}

1) Aromatic Polyamide Membranes

Aromatic Polyamide membranes are the most widely use membrane of conventional membrane structures. These membranes are used for both potable and industrial water production. The ultrathin polyamide film is formed on the surface of a thin microporous polysulfone support layer. The cohesion of the semipermeable thin film material with the microporous support layer is through interfacial polymerization of monomers containing polyamine and immersed in solvents containing a reactant to form a highly crosslinked combined structure 
[8]. PA membranes are preferred over CA membranes because they operate at lower pressures with higher productivity. PA membranes also allow lower salt passage than CA membranes. PA membranes have a negative charge when the $\mathrm{pH}$ level is greater than 5 . This electronegativity amplifies the co-ion repulsion and results in higher salt rejection. A disadvantage of PA membranes is when the systems $\mathrm{pH}$ level is below 4, the charge of the membrane changes to positive and the rejection of slat is reduced significantly. In the case of a system with $\mathrm{pH}$ levels below 4, CA membranes are more effective at salt rejection then PA membranes. The effective operating $\mathrm{pH}$ range for PA membranes is from 2 to 12, which is much wider than the 4 to $6 \mathrm{pH}$ range for CA membranes. This wider range allows ease of maintenance and cleaning of PA membranes. PA membranes are also non-biodegradable. Their usage life ranges from 5 to 7 years in comparison to a 3-to-5-year life usage range of CA membranes [8]. Unfortunately, PA membranes are highly susceptible to degradation by the oxidation of chorine and other oxidants. Chorine exposure can cause permanent damage to the thin-film structure which will result in significant reduction of salt rejection performance. Since most water processing systems use oxidants for biofouling control, the feed water to PA membranes must be dechlorinated prior to the RO separation stage.

2) Cellulose Acetate Membranes

The structure of CA membranes differs from PA membraned such that the top two layers are made of the same CA polymer but with different material characteristics. The entire thickness of a CA membrane is $100 \mu \mathrm{m}$ which is less than that of a PA membrane which is $160 \mu \mathrm{m}$. One important benefit of CA membranes is their surfaces have little charge and are usually considered as uncharged membranes. This makes CA membranes much less susceptible to cationic fouling [6]. CA membranes also have a smoother surface than PA membranes, further rendering their foul resistances. As mentioned before, one limitation of CA membranes is the small effective $\mathrm{pH}$ operating range. This is accompanied by a limiting operation temperature as well. CA membranes exposed to temperatures above $40^{\circ} \mathrm{C}$ caused compaction and lead to failure. To keep the $\mathrm{pH}$ levels in an effective operating range, feed water to the CA membranes must be reduced during normal plant operations resulting in additional processing cost. CA membranes also experience accelerated deterioration when exposed to microorganisms which can bioassimilate the membrane material. CA membranes, however, have a high tolerance to chorine which is often used to counter act the rate of destruction due to microbial activity [8]. The unique structure of CA membranes makes them denser than PA membranes. The high density leads to high head loss across CA membranes. Higher head loss across CA membrane system means higher operating pressures yielding increased operating cost. Since CA membranes have a high tolerance to oxide, they are ideal for municipal applications with high fouling potential. CA membrane's oxide tolerance also makes these membranes the superior choice when ultrapure water is needed such as for pharmaceutical and semiconductor industries [6]. 


\section{Membrane geometric configurations}

1) Hollow-Fiber

Until the mid-1990's, hollow-fiber elements were the most prevalent technology used for desalination. The semipermeable thin films described above are applied as a coating on the surface of a hollow fiber. These hollow fibers have and internal diameter of approximately $40 \mu \mathrm{m}$ and an outer diameter of approximately $80 \mu \mathrm{m}$. These fibers are bundled in sections with thicknesses ranging from 4 to 8 inches. These bundles of fibers are typically folded in half forming a single assembly with a length around 48 inches. The bundle of folded fibers is placed inside a cylindrical housing that is usually 6 to 12 inches in diameter. These housings are usually 50 to 54 inches long and epoxy sealed at both ends. One end of the element will contain the looped end of these fiber bundles created by the folding and the opposite end will have the open end of all the individual fibers. Saline feed water is pumped into the cylindrical housing and the water permeates through the membrane film coating into the inner side of the hollow tubes [8]. The salt and impurities are contained on the external side of the hollow-tubes while the permeate is collected and conveyed to a production collection output. This membrane configuration allows the largest semipermeable thin-film surface area exposure which makes them very suitable for high-salinity waters. Since the membrane surface area of a hollow-fiber membrane is so high the water flow regime is laminar. This low energy flow regime allows particulates and biofilms to easily attach along the membrane surface. Accumulation on the surface of these membrane structure leads to higher particulate fouling and biofouling [6]. The hollow fiber membranes offer a unique advantage in salt passage due to their high surface areas, but they require substantial amounts of pretreatments to keep them clean from accumulations.

2) Spiral-Wound

Today the desalination market is dominated by spiral-wound RO membrane elements. Spiral-wound membrane elements are typically made from approximately 40 individuals flat membrane sheets. These sheets are constructed form the triple-layered PA and CA membrane structures described before. These flat membrane sheets are assembled into membrane envelopes, each envelope consisting of two membrane sheets separated by a thin plastic net, referred to as a permeate spacers. The permeate spacers form a channel allowing evacuation of the permeate fluid upon separation from saline feed water. The membrane envelopes are sealed together on three of four sides [8]. The fourth side is left open to direct the permeate towards the central collection tube. The flat membrane envelopes are evenly spaced by plastic feed spacers. The feed spacers allow adequate flow and mixing of saline feed water though the entire length of the membrane element. The assembly of these flat-sheet membranes, and associated spacers, are wrapped around the perforated permeate collector tube to form a spiral-wound assembly. The assembly is maintained by wrapping the wound elements with tape and placing them in a fiberglass containment. Perforated plastic caps are then placed at the ends of each element. The end caps serve as 
longitudinal containment structures for the element structures and as seal carriers between membrane elements. They are perforated with specific patterns to optimize saline feed flow distribution. Endcaps of membrane elements are design to mate from one membrane element to the next, providing sufficient seals for multiple parallel membrane element configurations.

The typical configuration for commercial elements is seven elements per a vessel. Spiral-wound RO membranes are commercially available in sizes ranging from 2.5 inches in diameter to 19 inches in diameter. The most used commercial spiral-wound RO membrane size is 8 inches in diameter and 40 inches in length. These membrane elements have brine spacer thicknesses of 28 mils. The standard 8-in seawater element can produce anywhere from 3500 gal/day to 6500 gal/day. The standard 8-in brackish water element can produce anywhere from $7000 \mathrm{gal} /$ day to $10,000 \mathrm{gal} /$ day [6].

3) Flat-Sheet

Flat-sheet membrane elements are used in plate and frame RO systems. These systems consist of flat membrane envelopes made of two membrane sheets and a permeate spacer. The main difference between these element and spiral-wound elements is the membrane envelope is stacked flat one on top of another with feed water/brine spacer installed between each membrane envelope. The permeate spacers facilitate an envelope for permeate collection and prevent the membrane sheets form collapsing. The feed water/brine spacers facilitate feed water flow through the element.

Flat-sheet membrane elements have low membrane packing densities which make them significantly larger and more costly than conventional spiral-wound RO membrane elements. These elements are not widely utilized for municipal water RO desalination due to cost and size [6]. However, since these systems can be individually unpacked and cleaned, they have been used in food processing where high-solids applications are present.

\section{Membrane permeability}

1) Porous Membranes

The first type is that of the porous membrane and is shown in Figure 2. These membranes have a mean pore size diameter of $0.001-5 \mu \mathrm{m}$. With porous membranes, molecules move based on a pressure-driven convective flow through the tiny pores of the membrane, which are bigger than $10^{-9} \mathrm{~m}$. The different compound permeabilities is a result of the differences in steric hindrances between the components molecules and the membrane material. Within a porous membrane, the concentration of permeants is uniform, with the sole driving force across the membrane being the pressure gradient [9].

The vast majority of the membranes identified in prior research are made from prolypropylene $(\mathrm{PP})$, polyvinylidene fluoride (PVDF), polyte-trafluoroethylene (PTFE), polyethylene (PE) and polyethersulfone (PES). There are benefits to using this specific module, such as high mass transfer when non-wetted and lower thermal conductivity, as well as disadvantages, such as sensitivity to wetting, 


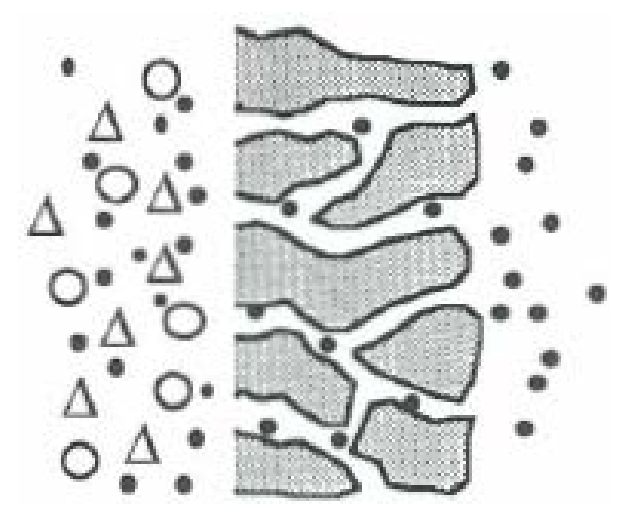

Figure 2. This diagram depicts the structure of a porous membrane.

scaling and intrapore salt precipitation, and capillary condensation. Figure 3 shows the basic structure of a porous membrane.

2) Non-Porous Membranes

The second type of membrane classified by the compounds of the liquid mixture being able to move through the membrane based on their force and the permeability of the membrane is that of the non-porous membrane and is shown in Figure 4.

Non-porous membranes are typically employed in the processes of reverse osmosis, nanofiltration, or molecular separation in the gas phase. With the non-porous membrane, the membrane is a dense film where permeants can diffuse through the membrane with pressure, concentration, or electrical potential gradient. The permeability as well as the selectivity is affected by the polymeric material. Differences in solubility and diffusivity allow for the separation process to occur. One downside of using a non-porous membrane is low flux, and accordingly, the dense film is typically constructed to be extremely thin and is deposited on top of asymmetric membranes [10].

3) Dense Membranes

The third type of membrane classified here is the dense membrane, which possesses a mean pore size diameter of less than $1 \mathrm{~nm}$. In the case of dense membranes, the molecules from the different compounds must first dissolve into the membrane matrix, whereby they can then diffuse through the membrane under a concentration gradient. It follows that the level of permeability of each species is determined by the solubility of each compound into the membrane material, which is the thermodynamic aspect, and by the rate at which each component diffuses through the membrane, which is the kinetic aspect. With the case of dense polymer membranes, the average pore diameter is within the thermal motion of the polymer chains, of which the membrane is constructed [9] (Figure $5)$.

With the process of desalination, salt is incapable of being vaporized, and it will therefore not cross the membrane. Accordingly, water vapor is the only compound which transfers across the membrane, like with the case of a porous membrane being used for membrane distillation applications. There are a few 


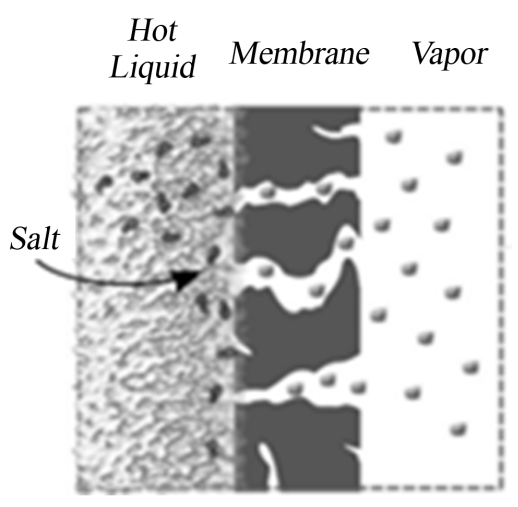

Figure 3. This diagram depicts the structure of a porous distillation membrane [11].

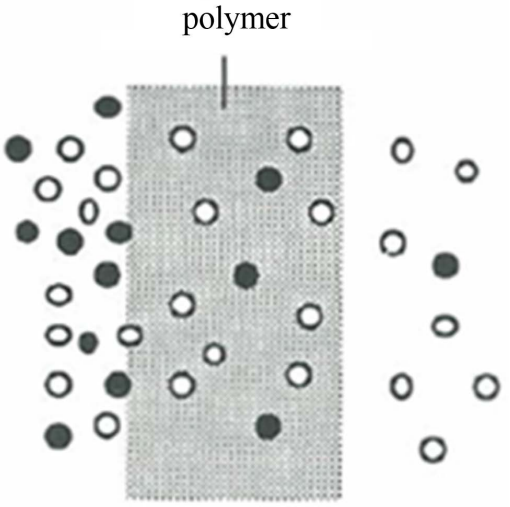

Figure 4. This diagram depicts the structure of a non-porous membrane [9].

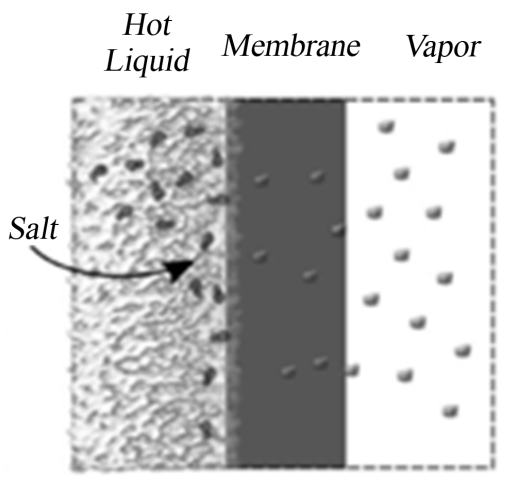

Figure 5. This diagram depicts the structure of a dense membrane [11].

benefits from applying this type of membrane, such as wetting protection, capillary condensation prevention particles, and the removal of salt. However, the disadvantages of using this type of membrane are increased thermal conductivity, unexplored concept, and issues with mechanical resistance [11].

Membranes are crucial for reverse osmosis purification systems to operate at their maximum potential and remove the most unwanted compounds. Most of the membranes in use at commercial reverse osmosis plants are made of cellulose acetate, polyamide, polysulfonate, and polyoxadiazole. There are typically 0.25 microns of skin and 100 microns of a support layer, which help in the mem- 
branes' abilities to filter out contaminants. When these membranes are made of cellulose acetate and polyamide, they possess strong salt rejection characteristics in the case of inorganic salts. However, in the case of organic salts, the rejection is observed to be lower and with a higher variability, showing a range of 0.3 to 0.96 [12] [13]. Membranes are usually rather pricey and can be expected to be of successful use for a long time. The best membranes demonstrate higher water flux (which means they are highly permeable to water) and ideally would allow for the flow of fairly large quantities of water through their bodies dependent on the total volume they occupy. The membrane should additionally be chemically, physically, and thermally stable in saline waters, and tough enough to sustain high pressures and fluctuations in the quality of the feed water.

\section{Motivation for Use of RO Systems}

Water scarcity is a worldwide and rapidly expanding crisis found in nearly every region of the world. As of today, roughly $36 \%$ of the global populous lives in water-scarce regions [14]. With a reverse osmosis system, the cleaning usually begins once the normalized flux drops $10 \%$ to $15 \%$, the normalized salt content of the permeate rises by $10 \%$, or when the pressure gradient in the pressure vessel drops by $15 \%$ [2]. With the membrane distillation module, the liquid that is at higher temperature in the feed is first evaporated at the liquid/vapor interface. Next, the resultant water vapor crosses the pores of the membrane. This vapor is a newly condensed compound on the permeate side. The principal force at work in this process of membrane distillation is the vapor pressure difference between the feed and the distillate. This is a product of the temperature and salt concentration within the layers adjacent to the membrane. While membrane distillation obviously holds great potential for its usage, there are some disadvantages that are a result of the operating conditions and the characteristics of the membrane. These disadvantages are the main factors which handicap the application of membrane distillation in industrial development and commercialization. The disadvantages are pore wetting, fouling, temperature and concentration polarization, and scaling [15]. However, all hope is not lost. There have been various innovations made to processes and materials which could potentially alleviate the problems resultant in the processes of membrane distillation [11]. Additionally, when attempting to optimize reverse osmosis desalination plants, the developers must choose the ideal membrane, which is of course dependent on the type of water. The selection of the appropriate membrane type is the most critical factor in determining the desalination plant's success. The goal of this review is to offer a detailed analysis on how to optimize water flux through the membrane by using the solution diffusion model.

\section{Models for Mass Transfer through Membranes}

There are assorted technologies which can be used to produce a generic separation process of a mixture, such as distillation, adsorption, absorption, or mem- 
branes. While each process possesses distinct transport equations, they can all still be used to separate a generic (A, B) mixture into A and B. A membrane is a selective barrier which permits the transport of certain components through its walls and captures others in the liquid or gas mixture. The stream which enters the membrane is known as the feed-stream, whereas the fluid that moves through the membrane is called the permeate. The fluid which holds the captured components is known as the retentate or the concentrate [9].

The identifying characteristic of membranes applied to separation applications is their ability to determine the permeation of varying species. There are two primary models most used to define this process of permeation. There is first the solution-diffusion model, in which permeants dissolve in the membrane material and then diffuse through the membrane down a concentration gradient. The different permeants observe a separation because of differences in the quantity of material which will dissolve in the membrane, along with the rate at which the material diffuses through the membrane. The second model is that of the pore flow model. In this model, permeants are separated by pressure-driven convective flow through tiny pores. The permeants separate since one of the permeants is excluded or filtered from some of the pores in the membrane through which other permeants move. Both of these models were first developed in the 1800s, however, the pore-flow model, being closer to normal physical experience, was more popular until the mid-1940s [1]. By 1980, the proponents of solution-diffusion became more popular. Today, there are not many modelers who employ the pore-flow model to observe reverse osmosis [1].

The overall mass balance over the membrane modules, assuming from the hypothesis that no chemical reaction occurs, is represented as follows [9]:

$$
\dot{n}_{f}=\dot{n}_{p}+\dot{n}_{r}=\sum_{i} \dot{n}_{f} x_{i . f}=\sum_{i} \dot{n}_{p} x_{i . p}+\sum_{i} \dot{n}_{r} x_{i . r}
$$

In (1), $\dot{n}_{f}$ represents the total molar flow-rate in the feed stream, $\dot{n}_{p}$ represents the total molar flow-rate in the permeate stream, $\dot{n}_{r}$ represents the total molar flow-rate in the retentate stream, and $x_{i}$ represents the molar fraction of component $i$.

When evaluating the overall mass balance of a single piece of membrane, as shown in Figure 6, and factoring in a control volume which encapsulates the entire membrane module, then the total mass flux across the membrane and into the mass balance can be written as follows [9]:

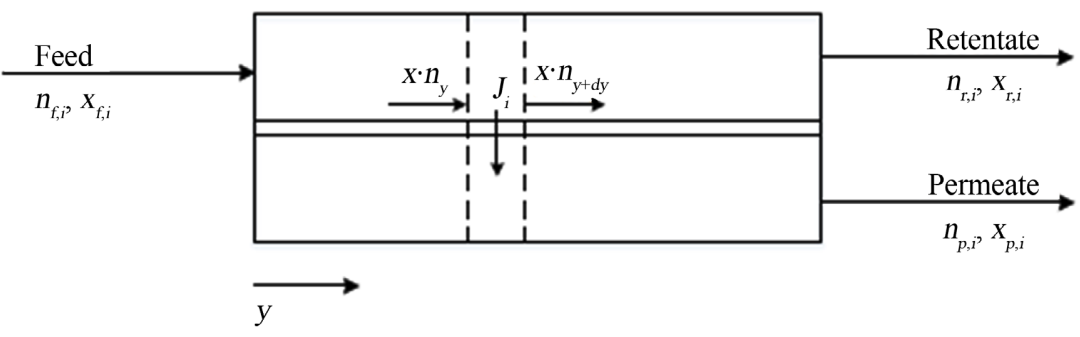

Figure 6. Graphical representation of the mass balance over the membrane module. 


$$
\dot{n}_{f}=\dot{n}_{r}+\dot{n}_{p}=\dot{n}_{r}+\int J \mathrm{~d} A
$$

In (2), $\int J$ represents the total molar flux of the permeating species, $J_{i}$ represents the molar flux equation for species, $i$, across the membrane, and, $A$, represents the membrane section area where mass transfer occurs.

Mass transport across membranes requires differing forces to propel its action. Non-equilibrium thermodynamics (NET) is the framework used to describe the wide range of behaviors which result from these disparate driving forces. NET employs four postulates above and beyond those of equilibrium thermodynamics: 1) The equilibrium thermodynamic relations apply to systems that are not in equilibrium, as long as gradients are not too large (local equilibrium); 2) All fluxes in the system may be written as linear relations involving all the driving forces; 3) No coupling of fluxes and forces occurs if the difference in tensorial order of the flux and force is an odd number (Curie's Postulate); 4) In the absence of magnetic fields, the matrix of the coefficients in the flux-force relations is symmetric (Onsager's reciprocal relations).

Mass transfer can be described when multiple driving forces are present. This is through the benefit of the Maxwell-Stefan Equation. The first calculation needed to process the Maxwell-Stefan equation is the entropy balance equation used to interpret irreversible processes (under the local equilibrium assumption, entropy remains a valuable state function in non-equilibrium). The Maxwell-Stefan Equation is the total summation of the terms for concentration, diffusion, pressure, force, and thermal diffusion. To simplify the Maxwell-Stefan Equation, the following constraints are found to be necessary: 1) Negligible curvature: Rcurv «1 (mass transport is unidirectional and perpendicular to the membrane surface); 2) Immobility of the matrix: $\left.V_{m}=0 ; 3\right)$ pseudosteady behavior: $\left(\partial c_{-}\right) / \partial t=0$ (diffusional terms within the membrane are short compared to those in the adjacent solution); 4) No thermal diffusion. The resultant Maxwell-Stefan Equation can be written thusly:

$$
\sum_{\beta=1}^{N} \frac{R T}{\mathrm{Ð}_{\alpha \beta} C}\left(x_{\beta} \bar{N}_{\alpha}-x_{\alpha} \bar{N}_{\beta}\right)=-x_{\alpha} R T \nabla \ln a_{\alpha}-z_{\alpha} F \nabla V+\left(\frac{\delta_{\alpha, m}}{\rho_{m}}-\bar{V}_{\alpha}\right) \nabla p
$$

In (3), $\bar{N}_{\alpha}$ and $\bar{N}_{\beta}$ represent the molar fluxes, $\delta_{\alpha, m}$ represent the Kronecker delta $\bar{V}_{\alpha}$, the partial molar volume of $\alpha . \boxplus_{\alpha \beta}$ is the Maxwell-Stefan diffusivities [9].

In the following sections, the model mass transfer for each module membrane distillation will be discussed.

\section{A. Model of mass transfer through porous membrane}

The porous network is what provides for the transport, whereas the membrane polymer itself is not instrumental in the transfer. With the case of porous membranes, there is an equilibrium present between the fluids on each side of the membrane and the material. This suggests a discontinuity in the concentration profiles at the interfaces. This equilibrium is governed by the solubility of these compounds in the polymer. 
The Darcy Equation is a useful tool for expressing the molar flux over the porous membrane. It can be written as follows:

$$
\bar{J}_{\alpha}=-\frac{\bigoplus_{\alpha \beta}}{R T} \bar{v}_{\alpha} c_{\alpha} \nabla p=-c_{\alpha} K^{\prime} \frac{\mathrm{d} p}{\mathrm{~d} z}
$$

In (4), $\frac{\mathrm{d} p}{\mathrm{~d} z}$ represents the pressure gradient, $c_{\alpha}$ represents the concentration of component $\mathrm{A}$ in the medium, and $K^{\prime}$ represents the permeability of the medium. To describe the mass transfer, a combination of Knudsen diffusion and Poiseuille flow can be used [16]. The membrane permeability coefficient $\wp$ is then calculated as follows:

$$
\wp=1.064 \frac{r_{p o r} \varepsilon}{\tau \delta}\left(\frac{M}{R T}\right)^{0.5}+0.125 \frac{r_{p o r}^{2} \varepsilon}{\tau \delta} \frac{M P_{p o r}}{\mu_{p o r} R T}
$$

In this equation, $r_{p o r}$ represents the radius of the pores $(\mathrm{m}), \mu$ represents the viscosity for the pore $(\mathrm{Pa} \cdot \mathrm{s}), R$ represents the gas constant $\left(\mathrm{J} \cdot \mathrm{K}^{-1} \cdot \mathrm{mol}^{-1}\right), P_{\text {por }}$ represents the membrane permeability for the pore $\left(\mathrm{kg} \cdot \mathrm{m}^{-2} \cdot \mathrm{s}^{-1} \cdot \mathrm{Pa}^{-1}\right), T$ represents the temperature (in Kelvin), \represents the membrane thickness (m), $M$ represents the molecular weight $\left(\mathrm{g} \cdot \mathrm{m}^{-1}\right), \tau$ represents the tortuosity, and $\varepsilon$ represents the porosity. This can also be referred to as the void fraction, which represents the void space in materials. Mathematically speaking, this is the ratio of the volume of interstices of a material to the volume of its mass. As we can see from (5), the permeability coefficient relies heavily on the properties of the membrane material: porosity $e$, membrane thickness $d$, tortuosity $s$ and pore diameter. In most cases, the pore diameter should be $0.3 \mathrm{~lm}$ or smaller in order to avoid wetting. Additionally, the optimal membrane thickness is known to be between 20 and $200 \mu \mathrm{m}$. The slimmer the membrane, the higher the flux, in spite of the fact that a thinner membrane could cause higher loss of heat via conduction. The porosity should be as high as possible; $e>75 \%$; which will ensure a high insulation, and thus a high thermal efficiency. For this case, a lower tortuosity is recommended, something around 1.1 to 1.2. When a high porosity is attained, the tortuosity is assumed to be sufficiently low. In order to avoid wetting, the adequate LEP is around 2:5 bar. This value is based on the material properties and the hydrophobic character of the membrane [17]. A contact angle of roughly 120 degrees is recommended to ensure the hydrophobicity of the membrane [11].

\section{B. Model of mass transport through dense membrane and non-porous membrane}

In the case of a dense and non-porous membrane, a solution-diffusion process produces the separation, and it requires the sorption of the compound at the upstream interface of the membrane, followed by its diffusion through the material, and then its desorption downstream of the membrane. Water permeability is determined by two factors according to the solution-diffusion mechanism. First, there is the solubility $(S)$, which relies on the interaction of the polymer matrix with water. Then, there is diffusivity $(D)$, which is primarily determined by the 
diameter of the components to be separated and by the mobility of the polymer chains [11]:

$$
P=D S
$$

The concentration gradient supplies the driving force of transport through a dense membrane, and in the case of membrane distillation, this concentration gradient creates a variance in vapor pressure on both sides of the membrane. In order to facilitate transport, a lower permeate vapor pressure than that of the feed side must be maintained [11]. The mass transfer resistance present in a self-supporting dense membrane is always much higher than that of a dry microporous membrane of the same thickness. Because there is an absence of porosity, the effective diffusion coefficients of the transported molecules are many orders of magnitude lower than those observed in a microporous membrane. Because of this rule, the thickness of the dense material must be brought to a very low value so that competition with the performances of dry microporous membranes can be achieved.

\section{The Solution Diffusion Model}

When it comes to transport models for permeation in a polymer membrane, the solution-diffusion model has gained the most traction amongst researchers and engineers. This model provides for the following membrane separation processes: reverse osmosis, gas separation, and pervaporation [1].

Transport can only happen through diffusion in the solution-diffusion model. For it to be successfully transported, the component must dissolve in the membrane first [11]. Within the solution-diffusion model, the pressure within the membrane does not change at the high-pressure value (pr) and is thus taken as constant, and the gradient in chemical potential across the membrane is expressed as a smooth gradient in solvent activity. This can be seen in Figure 7. In the solution-diffusion model, the permeants dissolve (sorption) in the membrane material at the upstream interface when a concentration gradient in

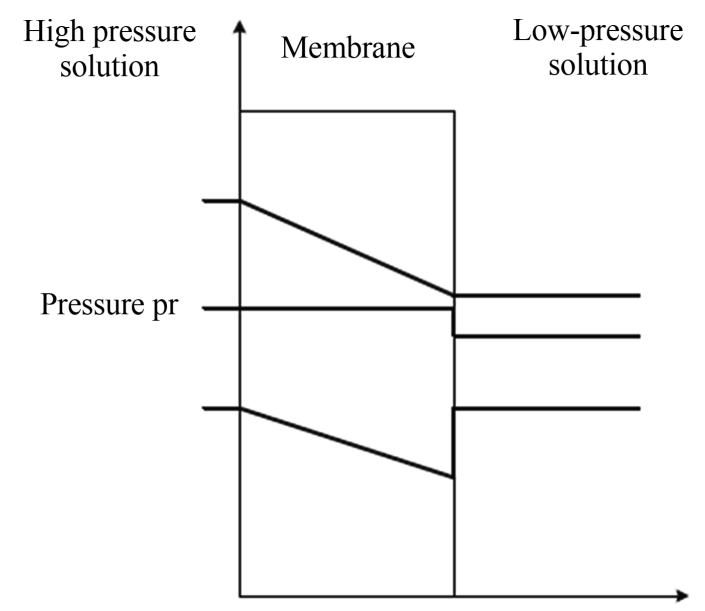

Figure 7. This diagram depicts the solution-diffusion permeation model for mass transfer through the membrane. 
present that permits permeants to diffuse through the membrane and be desorbed on the downstream interface side. These different permeants will separate due to the fact that each material has a different diffusion rate in the membrane [18].

When we return to the Maxwell Stephen Equation (3), and assume that no pressure gradient exists within the membrane and no forces act on the membrane, the molar flux of the component can be written as follows:

$$
\bar{J}_{\alpha}=-c \bigoplus_{\alpha \beta} x_{\alpha} \nabla \ln a_{\alpha}
$$

Additionally, when we introduce the definition of activity in accordance with the hypothesis of perpendicular flux, the Fick Equation can be seen below [9]:

$$
\bar{J}_{\alpha}=-c \bigoplus_{\alpha \beta} x_{\alpha} \nabla x_{\alpha}=-c D_{\alpha, \beta} \frac{\mathrm{d} x_{\alpha}}{\mathrm{d} z} \hat{n}
$$

Here, $\bigoplus_{\alpha \beta}=D_{\alpha, \beta}$ in an ideal mixture.

With the framework of the solution-diffusion model, the goal is to connect the flux equation and the continuity equation to the conditions in the mixture bathing the membrane. The continuity equation is represented mathematically below [9]:

$$
c \frac{D x_{\alpha}}{D t}=-\nabla \cdot \bar{J}_{\alpha}+\left(R_{\alpha}-x_{\alpha} \sum_{\beta}^{N} R_{\beta}\right)
$$

Thus, it's necessary to consider the conditions at the membrane interfaces: equilibrium assumption between the membrane surface and the mixture on the retentate or permeate of the membrane has been made [9]. In examining the flux equations of Fick and Darcy, and substituting them into the continuity equation, the solution-diffusion model can be expressed mathematically as follows [9]:

$$
\frac{\mathrm{d}}{\mathrm{d} z}\left(c D_{\alpha, \beta} \frac{\mathrm{d} x_{\alpha}}{\mathrm{d} z}\right)=0 \text { Solution-Diffusion Model }
$$

The following boundary conditions are applied to solve the solution-diffusion model: At $z=0, \quad x_{\alpha}=x_{\alpha, R}^{I}$ and $z=L, x_{\alpha}=x_{\alpha, P}^{I}$ and yields:

$$
J_{\alpha}=c D_{\alpha, \beta} \frac{x_{\alpha, R}^{I}-x_{\alpha, P}^{I}}{\delta}
$$

In this case $x_{\alpha, R}^{I}, \quad x_{\alpha, P}^{I}, \quad c_{\alpha, R}^{I}, \quad c_{\alpha, P}^{I}$ represent molar fractions and compositions for components within the membrane, but either at the feed or permeate interface, and membrane thickness. The values of $x_{\alpha, R}^{I}, \quad x_{\alpha, P}^{I}$ can be seen in (12) and (13) [9]:

$$
\begin{gathered}
x_{\alpha, r}^{I}=x_{\alpha, r} \frac{\gamma_{\alpha, r}}{\gamma_{\alpha, r}^{I}} \\
x_{\alpha, p}^{I}=x_{\alpha, p} \frac{\gamma_{\alpha, p}}{\gamma_{\alpha, p}^{I}}\left\{\exp \left[-\frac{\tilde{v}_{\alpha, l}}{R T}\left(p_{r}-p_{p}\right)\right]\right\}
\end{gathered}
$$

When we substitute these values in the solved solution-diffusion model and assume that the ratio between the activity coefficient is the same at the retentate 
or at the permeate side, this will yield the following arrangement [9]:

$$
J_{\alpha} \approx \frac{c D_{\alpha, \beta}}{\delta} \frac{\gamma_{\alpha, r}}{\gamma_{\alpha, r}^{I}}\left\{x_{\alpha, r}-x_{\alpha, p} \exp \left[-\frac{\tilde{v}_{\alpha, l}}{R T}\left(p_{r}-p_{p}\right)\right]\right\}
$$

Here, the subscripts $r$ and $p$ identify the retentate and permeate conditions, respectively. In the solved solution diffusion model, the results confirmed that flux rate is proportional to a gradient in the chemical potential and pressure drop. As previously stated, reverse osmosis is primarily employed for the desalination of saltwater. In this arrangement, membranes that are permeable to water yet impermeable to salt are exploited. The objective of water desalination is the production of fresh water at the permeate side. In Figure 8, the schematic of a membrane module for water desalination is presented. Because a pressure difference is administered across the membrane, a liquid mixture at both sides can be expected. Pressurized water which possesses dissolved salts contacts the feed side of the membrane. At the same time, water that has had its salt removed is withdrawn as a low-pressure permeate. This section seeks to express the flux equations in terms of the pressure gradient through the membrane and render them linear.

It is possible to rewrite (14) in terms of the change from the molar fraction to the concentration below:

$$
J_{w}=\frac{Q_{w}}{\delta} X_{w, f} \frac{\tilde{v}_{w}}{R T}\left[\left(p_{f}-p_{p}\right)-\Delta \pi\right]
$$

In looking at (15) and considering the $\Delta \pi=p_{r}-p_{p}$, three potential scenarios can be drawn for a dense solution-diffusion membrane:

1) $p_{r}-p<\Delta \pi . J_{w}<0$. Normal osmosis takes place. The water flows from the pure-water side to the sea-water side, as seen in Figure 9.

2) $p_{r}-p=\Delta \pi, J_{w}=0$. Here, the osmotic equilibrium is reached, and no flux occurs due to the fact that the osmotic pressure is counterbalanced by the pressure difference, as seen in Figure 10.

3) $p_{r}-p>\Delta \pi, J_{w}>0$. Reverse osmosis takes place. The water flows from the sea-water side to the pure-water side, as seen in Figure 11.

\section{Thin, Highly Permeable Membrane}

It is worth noting that recent studies and prior research have affirmed the possibility of creating such a thin, highly permeable membrane, such as the study

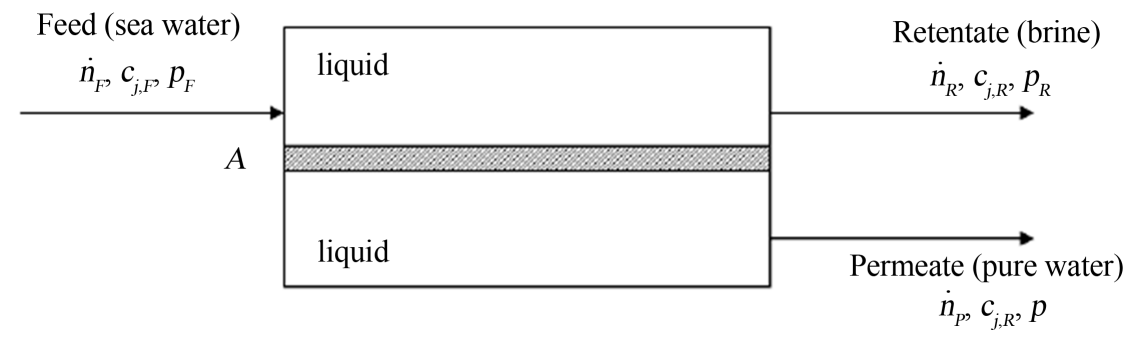

Figure 8. Schematic of a membrane module for reverse osmosis [19]. 


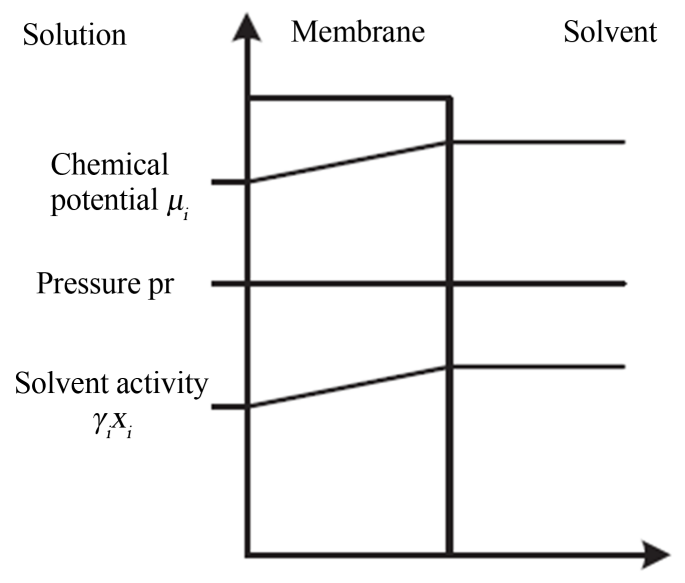

Figure 9. Schematic of an osmosis solution-diffusion membrane [9].

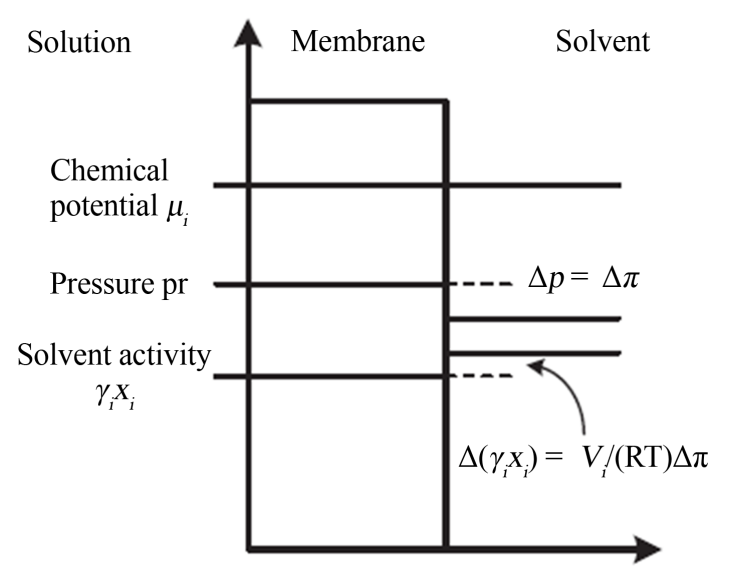

Figure 10. Schematic of an osmotic equilibrium solution-diffusion membrane [9].

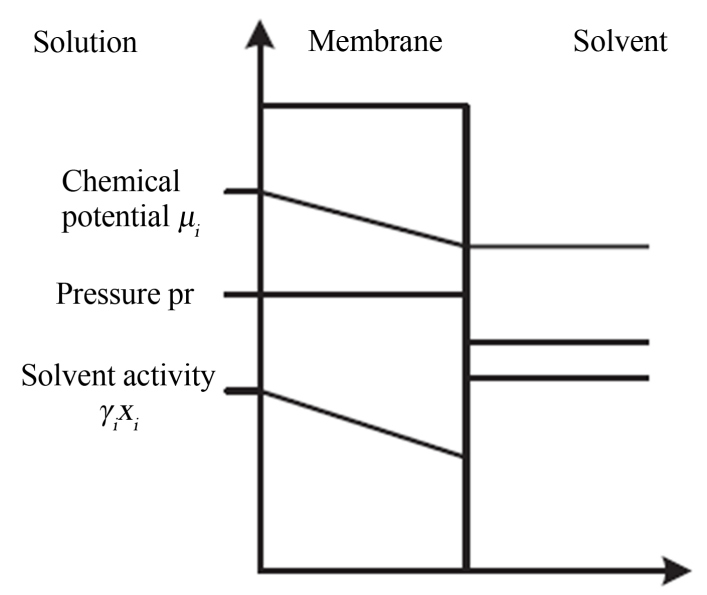

Figure 11. Schematic of reverse osmosis solution-diffusion membrane [20].

done by Soyekwo in 2017 where a cellulosic composite membrane with a thickness of 64 to $111 \mathrm{~nm}$ was synthesized [21]. A smooth, dense, ultrathin PEI barrier was detected on the surface of this cellulosic membrane that had an estimated thickness of around $10 \mathrm{~nm}$. Further, another study revealed that several 
nanometric thin films were fabricated using block copolymers, PEO and PBT. The thickness of the membranes varied between 80 and $500 \mathrm{~nm}$. These membranes were applied to the $\mathrm{CO} 2$ absorption, finding a higher permeability than commercial membranes [22]. Self-standing nanofilms of polysulfones doped with sulfonate polysulfones were also prepared via solvent evaporation obtaining a self-standing film of $46 \mathrm{~nm}$ thickness [23]. In another study issued by Karan et al. in 2015, $10 \mathrm{~nm}$-thick polyamide membranes were produced using controlled interfacial polymerization, and it was found that the nanofilms were sufficiently rigid [24].

\section{Practical Application of the Solution-Diffusion Model}

A study performed by Maddah and Almugahwi in 2017, applied the diffusion-solution model to determine the appropriate membrane for a RO plant [18]. This study provides an example of the practical application of this model for plant optimization. With water-salt solution, the reverse osmosis permeation expression used in the study can be simplified and easily be related to the previous equation (15). The specific equations for this study are (16) and (17) [1] [25]:

$$
\begin{gathered}
J_{i}=A(\Delta p-\Delta \pi) \\
A=\frac{P_{i} c_{i o} v_{i}}{R T l}
\end{gathered}
$$

In (16), $J_{i}$ is the membrane flux component i for water in (gfd), $\Delta p$ is the applied pressure drop across the membrane in (psi), $\Delta \pi$ is the osmotic pressure drop across the membrane in (psi), $A$ is the water permeability constant in $\left(\mathrm{cm} / \mathrm{atm}^{*} \mathrm{~s}\right)$. In (17), $P_{i}$ is the permeability of component $\mathrm{i}$ in water in $\left(\mathrm{cm}^{2} / \mathrm{s}\right), c_{i o}$ is the initial mole concentration of water in (ppm), $v_{i}$ is the water molar volume in $\left(\mathrm{cm}^{3} / \mathrm{mol}\right), T$ is the water temperature in $(\mathrm{K}), R$ is the gas constant $\left(\mathrm{m}^{3} \mathrm{~atm} /\right.$ $\operatorname{mol}^{\star} \mathrm{K}$ ), and $l$ is the membrane thickness which is assumed to be similar to the spacer thickness in (mil).

Table 1 provides the data from the Abqaiq 500 RO plant applied to determine the values for osmotic pressure drop for the RO membrane from (16) and (17). To calculate the osmotic pressure for seawater sources, the same information of Shedgum/Abqaiq groundwater was applied at Abqaiq $500 \mathrm{RO}$ plant, except for flux and salinity values, for the treatment of either Arabian Gulf or Red Sea waters [25]. Water permeability was determined to be approximately $9.5 \times 10^{-7}$ $\mathrm{cm}^{2} / \mathrm{s}$ [28]. Membrane resistance constant for each BWRO Toray membrane can be determined by using (18) below [28].

$$
J_{i}=\frac{\Delta p}{k R_{m}}
$$

In (18), $k$ is the dynamic viscosity of water in $\left(\mathrm{lb}^{\star} \mathrm{s} / \mathrm{ft}^{2}\right)$ and $R_{m}$ is the membrane resistance in $\left(\mathrm{t}^{-1}\right)$. The Van't Hoff [29] osmotic pressure $(\pi)$ formula is useful for estimating the osmotic pressure of an aqueous solution from its molar 
Table 1. Data of RO membrane process at abqaiq 500 plant and the two seawater studied scenarios [1] [26] [27].

\begin{tabular}{|c|c|c|c|}
\hline Parameter & $\begin{array}{c}\text { Shedgum/Abqaiq } \\
\text { Groundwater }\end{array}$ & $\begin{array}{c}\text { Arabian Gulf } \\
\text { Water Scenario }\end{array}$ & $\begin{array}{c}\text { Red Sea } \\
\text { Water Scenario }\end{array}$ \\
\hline Membrane type & \multicolumn{3}{|c|}{ Toray TM720D-400 with 8" } \\
\hline RO Module & \multicolumn{3}{|c|}{72 parallel membranes $\times 8$ units } \\
\hline Membrane thickness $(\ell)$ & \multicolumn{3}{|c|}{ Assumed to be like spacer thickness of $34 \mathrm{mil}$} \\
\hline Membrane area (Area) & \multicolumn{3}{|c|}{$400 \mathrm{ft}^{2}$} \\
\hline $\begin{array}{l}\text { Max pressure drop } \\
\text { per vessel }(\Delta P)\end{array}$ & \multicolumn{3}{|c|}{$\sim 60$ psi } \\
\hline $\begin{array}{l}\text { Max pressure drop } \\
\text { per membrane }(\Delta P)\end{array}$ & \multicolumn{3}{|c|}{$\sim 20 \mathrm{psi}$} \\
\hline Water salinity $\left(c_{i o}\right)$ & $\sim 2800 \mathrm{ppm}$ & $\sim 41,070 \mathrm{ppm}$ & $\sim 42,070 \mathrm{ppm}$ \\
\hline Membrane water flux $\left(J_{i}\right)$ & $\sim 18$ gfd & $\sim 12$ gfd & $\sim 12$ gfd \\
\hline Water temperature $(T)$ & \multicolumn{3}{|c|}{$\sim 25^{\circ} \mathrm{C}$} \\
\hline Water permeability constant $\left(P_{i}\right)$ & \multicolumn{3}{|c|}{$9.5 \times 10^{-7} \mathrm{~cm}^{2} / \mathrm{s}$} \\
\hline Water molar volume $\left(v_{i}\right)$ & \multicolumn{3}{|c|}{$18 \mathrm{~cm}^{3} / \mathrm{mol}$} \\
\hline Gas constant $(R)$ & \multicolumn{3}{|c|}{$8.2057 \times 10^{-5} \mathrm{~m}^{3} \mathrm{~atm} / \mathrm{mol} \mathrm{K}$} \\
\hline
\end{tabular}

concentrations of dissolved species. In the three various water sources in (19), the overall required osmotic pressure $\operatorname{drop}(\Delta \pi)$ for a water treatment plant was explored [18].

$$
\pi=\mathcal{M} T
$$

In (19), $\mathcal{M}$ is the molar concentration of dissolved species in $(\mathrm{mol} / \mathrm{L}), \mathbb{R}$ is the Ideal gas constant, $0.08206 \mathrm{~L}$ atm/mol K. Equation (20) can be used to determine the fitness of a membrane in being able to separate salt from the feed solution. This is known as membrane removal percentage and represented by, $\chi$. This fitness increases with the applied pressure. Three sources from Table 1 derive the feed TDS concentration, while the outlet TDS concentration can be calculated using (20). The water molecular weight of $18(\mathrm{~g} / \mathrm{mol})$ is useful in converting our ppm values to molar concentrations of TDS [28].

$$
X=\left(\frac{c_{j o}-c_{j l}}{c_{j o}}\right) * 100
$$

In (20), $X$ is the membrane removal percentage, $c_{j o}$ is the initial mole concentration of component, $j$, which is salt in (ppm), and $c_{j l}$ is the final mole concentration of component $j$ in (ppm).

Altaee's study demonstrates how permeate flow, pressure, and recovery rate are distributed in a manner very similar to membranes per RO vessel [30]. Additionally, another field study proved a stronger performance by rearranging the elements in pressure vessels so that the pressure drop and permeate conductivity 
across the vessel can be reduced [31]. The traditional flux rates and maximum recovery values for the groundwater and the two studied water source scenarios (Arabian Gulf and Red Sea waters) at Abqaiq 500 RO plant are on display in Table 2.

The applied pressure drop and suggested flux values are calculated for each of the different types of membranes. The same osmotic pressure drop for each case is used to determine the results of different Toray BWRO membrane types at high, low, and standard operating pressures. The applied pressure drop should be greater than the calculated osmotic pressure in order to create a positive flux [18].

(16) and (17) permitted the calculation of the osmotic pressure drop $(\Delta \pi)$ for each water source. These calculations are evidenced in Table 3, where the osmotic pressure of the groundwater source is lower than Arabian Gulf and Red Sea water sources. This is due to the flux rates and water salinity. The flux rates for Arabian Gulf and Red Sea waters are approximately half that of the groundwater source, but water salinity of the groundwater source is significantly lower than the other sources. It follows that the required applied pressure drop must be greater in the case of seawater sources. This is because of the higher determined osmotic pressure values of these sources. Because the plant configuration has 8 elements per vessel, we should have a maximum osmotic pressure of 60 psi or less per vessel. This is equivalent to a max pressure of 7.5 psi per membrane if the pressure is distributed equally on membranes per vessel. The selected applied pressure range for this analysis is 6.5 to 7.5 psi. The maximum pressure values are assigned to the different membranes' dependent on their category.

Maddah and Almugahwi in their study find the relationship between the applied pressure drops and the overall water flux rates for the groundwater source.

Table 2. Characteristics of groundwater sources and studied water sources at Abqaiq 500 RO plant.

\begin{tabular}{cccc}
\hline Water Source & $\begin{array}{c}\text { Shedgum/Abqaiq } \\
\text { Groundwater }\end{array}$ & $\begin{array}{c}\text { Arabian } \\
\text { Gulf }\end{array}$ & Red Sea \\
\hline Feed silt density index & SDI $<3$ & SDI $<4$ & SDI $<4$ \\
Typical target flux, gfd & 18 & 12 & 12 \\
Max. element recovery, \% & 19 & 14 & 14 \\
\hline
\end{tabular}

Table 3. Calculated osmotic pressure drop $(\Delta \pi)$ for each water source form (16) and (17), $[18]$.

\begin{tabular}{ccccccc}
\hline Water Source & $\begin{array}{c}\mathrm{A} \\
(\mathrm{cm} / \mathrm{atms})\end{array}$ & $\begin{array}{c}J_{i} \\
(\mathrm{~cm} / \mathrm{s})\end{array}$ & $\begin{array}{c}J_{i} / \mathrm{A} \\
(\mathrm{atm})\end{array}$ & $\begin{array}{c}\Delta \pi \\
(\mathrm{atm})\end{array}$ & $\begin{array}{c}\Delta \pi \\
(\mathrm{psi})\end{array}$ & $\begin{array}{c}\Delta \pi \text { per } \\
\text { vessel }<60(\mathrm{psi})\end{array}$ \\
\hline $\begin{array}{c}\text { Shedgum/Abqaiq } \\
\text { groundwater }\end{array}$ & 0.00808 & 0.00083 & 0.10288 & 0.441 & 6.48 & 51.84 \\
$\begin{array}{c}\text { Arabian Gulf water } \\
\text { Red Seawater }\end{array}$ & 0.00755 & 0.00056 & 0.07417 & 0.470 & 6.90 & 55.21 \\
\hline
\end{tabular}


They find that the maximum possible flux for the groundwater in the standard membranes is around $11 \mathrm{gfd}$. The greatest groundwater flux that can be obtained is $8 \mathrm{gfd}$ at the high-pressure and low-membranes thickness. Also, the maximum flux observed for low-pressure membranes is nearly $15 \mathrm{gfd}$. This observation is relative to the membrane thickness, where the smallest membrane thickness (28 $\mathrm{mm}$ ) was able to produce the highest flux. This finding proves that an inverse relationship exists between the membrane thickness and the water flux rate, and a linear relation between the applied pressure drop and the overall water flux can be realized as well [18]. Finally, the membrane resistance for the three water types is investigated. The study shows that seawater sources have greater membrane resistances than groundwater sources. This is due to having lower flux and higher TDS. The highest membrane resistance is obtained because of its low-pressure category, and it has the greatest membrane thickness of $31 \mathrm{~mm}$ [18].

\section{Conclusions}

The solution-diffusion model can be applied to determine the optimum operational parameters of a variety of membranes. As seen in the example of the Abqaiq plant, the osmotic pressure of an array of Toray membranes was evaluated for the optimum configuration. The osmotic pressure values were calculated for Arabian Gulf and Red Sea waters to estimate flux rates in the membranes for use with saline water.

A linear relationship can be seen to exist between the water flux and the applied pressure drops, and thus it is confirmed that membrane flux decreases with the increase in membrane thickness when the pressure drop is constant. The findings from the Abqaiq $500 \mathrm{RO}$ plant examples show that the lowest membrane resistance and the highest overall water flux are the best membrane to select. Maddah and Almugahwi example reveals the effectiveness of the use of the solution-diffusion mode for determining optimal membrane parameters. These findings reveal the effectiveness of the use of the solution-diffusion model for determining optimal membrane parameters. Ultimately, the design of more effective membrane parameters will mean that RO and other membrane-based desalination systems can expect longer operational lives with fewer membrane-based concerns, like scaling or fouling. Future research endeavors should be lent towards exploring new ways to prolong membrane life, along with additional exploration into the effects that solvents have on membrane health.

\section{Conflicts of Interest}

The authors declare no conflicts of interest regarding the publication of this paper.

\section{References}

[1] Wijmans, J. and Baker, R. (1995) The Solution-Diffusion Model: A Review. Journal of Membrane Science, 107, 1-21. https://doi.org/10.1016/0376-7388(95)00102-I 
[2] Ahuchaogu, A.A., Chukwu, O.J., Obike, A.I., Igara, C.E., Nnorom, I.C. and Echeme, J.B.O. (2018) Reverse Osmosis Technology, Its Applications and Nano-Enabled Membrane. International Journal of Advanced Research in Chemical Science, 5, 20-26. https://doi.org/10.20431/2349-0403.0502005

[3] Woodard, J. (2020) What Is a Reverse Osmosis System and How Does It Work? https://www.freshwatersystems.com/blogs/blog/what-is-reverse-osmosis

[4] Letcher, T. (2012) Comprehensive Renewable Energy. Newnes, London.

[5] Garud, R.M., Kore, S.V., Kore, V.S. and Kulkarni, G.S. (2011) A Short Review on Process and Applications of Reverse Osmosis. Universal Journal of Environmental Research \& Technology, 1, 233-238.

[6] Voutchkov, N. (2013) Desalination Engineering Planning and Design. McGraw-Hill, New York.

https://www.academia.edu/31057784/Desalination Engineering_Planning and Des ign pdf

[7] Nastase, C., Nastase, F., Dumitru, A., Ionescu, M. and Stamatin, I. (2005) Thin Film Composites of Nanocarbons-Polyaniline Obtained by Plasma Polymerization Technique. Composites Part A: Applied Science and Manufacturing, 36, 481-485. https://doi.org/10.1016/j.compositesa.2004.10.009

[8] Jamaly, S., Darwish, N.N., Ahmed, I. and Hasan, S.W. (2014) A Short Review on Reverse Osmosis Pretreatment Technologies. Desalination, 354, 30-38. https://doi.org/10.1016/j.desal.2014.09.017

[9] Gazzani, M., Mazzotti, M., Milella, F. and Gabrielli, P. (2016) Membrane Separations-Rate Controlled Separation Processes. ETH Zürich, Zürich.

[10] Filtration, S. (2014) Polymeric Membranes: Porous vs. Non-Porous. https://synderfiltration.com/learning-center/articles/introduction-to-membranes/p olymeric-membranes-porous-non-porous/

[11] Mejia Mendez, D., Castel, C., Lemaitre, C. and Favre, E. (2018) Membrane Distillation (MD) Processes for Water Desalination Applications. Can Dense Selfstanding Membranes Compete with Microporous Hydrophobic Materials? Chemical Engineering Science, 188, 84-96. https://doi.org/10.1016/j.ces.2018.05.025

[12] Pozderović, A., Moslavac, T. and Pichler, A. (2006) Concentration of Aqua Solutions of Organic Components by Reverse Osmosis. I: Influence of Trans-Membrane Pressure and Membrane Type on Concentration of Different Ester and Aldehyde Solutions by Reverse Osmosis. Journal of Food Engineering, 76, 387-395. https://doi.org/10.1016/j.jfoodeng.2005.05.038

[13] Senthilmurugan, S. and Gupta, S.K. (2006) Separation of Inorganic and Organic Compounds by Using a Radial Flow Hollow-Fiber Reverse Osmosis Module. Desalination, 196, 221-236. https://doi.org/10.1016/j.desal.2006.02.001

[14] Guerquin, F. (2020) Land Degradation Neutrality for Briefing Note Water Security and Combatting Drought. Food and Agriculture Organization, Rome. https://www.fao.org/3/ca7468en/CA7468EN.pdf

[15] Khayet, M. and Matsuura, T. (2011) Membrane Distillation: Principles and Applications. Elsevier, Amsterdam. https://doi.org/10.1016/C2009-0-17487-1

[16] Ibrahim, S. and Alsalhy, Q. (2012) Modeling and Simulation for Direct Contact Membrane Distillation in Hollow Fiber Modules. AIChE Journal, 59, 589-603. https://doi.org/10.1002/aic.13845

[17] Pangarkar, B., Deshmukh, S., Sapkal, V. and Sapkal, R. (2014) Review of Membrane 
Distillation Process for Water Purification. Desalination and Water Treatment, 57, 2959-2981. https://doi.org/10.1080/19443994.2014.985728

[18] Maddah, M. and Almughwi, H.A. (2017) Application of the Solution-Diffusion model to Optimize Water flux in Reverse Osmosis Desalination Plants. AWWA/ AMTA Membrane Technology Conference and Exposition 2017, Long Beach.

[19] Baker, R.W. (2012) Membrane Technology and Applications. 3rd Edition, Wiley, Hoboken. https://doi.org/10.1002/9781118359686

[20] Luis, P. and van der Bruggen, B. (2015) Pervaporation Modeling: State of the Art and Future Trends. In: Basile, A., Figoli, A. and Khayet, M., Eds., Pervaporation, Vapour Permeation and Membrane Distillation, Woodhead Publishing, Sawston, 87-106. https://doi.org/10.1016/B978-1-78242-246-4.00004-0

[21] Soyekwo, F., Zhang, Q., Gao, R., Qu, Y., Lin, C., Huang, X., et al. (2017) Cellulose Nanofiber Intermediary to Fabricate Highly-Permeable Ultrathin Nanofiltration Membranes for Fast Water Purification. Journal of Membrane Science, 524, 174-185. https://doi.org/10.1016/j.memsci.2016.11.019

[22] Xu, C.H., Chen, X., Liu, Y.J., Xie, B., Han, M., Song, F.Q. and Wang, G.H. (2010) Enhanced Thermal Stability of Monodispersed Silver Cluster Arrays Assembled on Block Copolymer Scaffolds. Nanotechnology, 21, Article ID: 195304. https://doi.org/10.1088/0957-4484/21/19/195304

[23] Yuan, H.G., Liu, Y.Y., Liu, T.Y. and Wang, X.L. (2017) Self-Standing Nanofilms of Polysulfone Doped with Sulfonated Polysulfone via Solvent Evaporation for Forward Osmosis. Journal of Membrane Science, 523, 567-575. https://doi.org/10.1016/j.memsci.2016.09.034

[24] Karan, S., Jiang, Z. and Livingston, A. (2015) Sub-10 nm Polyamide Nanofilms with Ultrafast Solvent Transport for Molecular Separation. Science, 348, 1347-1351. https://doi.org/10.1126/science.aaa5058

[25] Sagle, A. and Freeman, B. (2004) Fundamentals of Membranes for Water Treatment. The Future of Desalination in Texas, 2, Article No. 137.

[26] Abdel-Aal, E.A., Farid, M.E., Hassan, F.S. and Mohamed, A.E. (2015) Desalination of Red Sea Water Using Both Electrodialysis and Reverse Osmosis as Complementary Methods. Egyptian Journal of Petroleum, 24, 71-75. https://doi.org/10.1016/j.ejpe.2015.02.007

[27] Smith, R., Purnama, A. and Al-Barwani, H. (2007) Sensitivity of Hypersaline Arabian Gulf to Seawater Desalination Plants. Applied Mathematical Modelling, 31, 2347-2354. https://doi.org/10.1016/j.apm.2006.09.010

[28] Crittenden, J.C., Trussell, R.R., Hand, D.W., Howe, K. and Tchobanoglous, G. (2012) MWH's Water Treatment: Principles and Design. John Wiley \& Sons, Hoboken. https://doi.org/10.1002/9781118131473

[29] Prausnitz, J.M., Lichtenthaler, R.N. and Azevedo, E.G. (1999) Molecular Thermodynamics of Fluid-Phase Equilibria. Prentice Hall Inc., Hoboken.

[30] Altaee, A. (2012) A Computational Model to Estimate the Performance of 8 Inches RO Membranes in Pressure vessel. Journal of Membrane and Separation Technology, 1, 60-71. https://doi.org/10.6000/1929-6037.2012.01.01.8

[31] Madaeni, S.S., Afshar, M., Jaafarzadeh, N., Tarkian, F. and Ghasemipanah, K. (2011) Rearrangement of Membrane Elements in the Pressure Vessels for Optimum Utilization of Reverse Osmosis Process. Chemical Engineering Research and Design, 89, 48-54. https://doi.org/10.1016/j.cherd.2010.04.021 